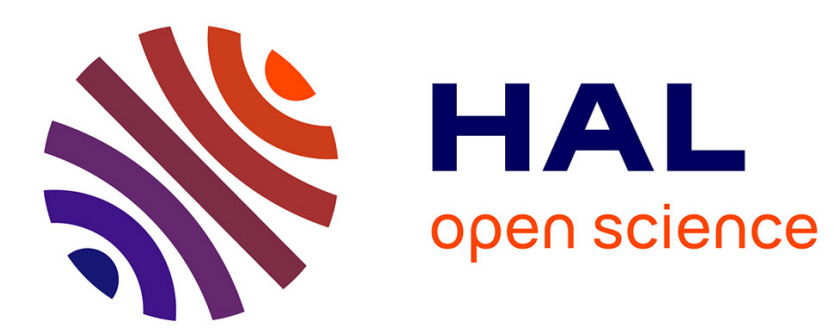

\title{
Displacement dependent pressure load for finite deflection of doubly-curved thick shells and plates
}

Marco Amabili, Ivan Breslavsky

\section{To cite this version:}

Marco Amabili, Ivan Breslavsky. Displacement dependent pressure load for finite deflection of doublycurved thick shells and plates. International Journal of Non-Linear Mechanics, 2015, 77, pp.265-273. 10.1016/j.ijnonlinmec.2015.09.007 . hal-01303327

\section{HAL Id: hal-01303327 https://hal.science/hal-01303327}

Submitted on 18 Apr 2016

HAL is a multi-disciplinary open access archive for the deposit and dissemination of scientific research documents, whether they are published or not. The documents may come from teaching and research institutions in France or abroad, or from public or private research centers.
L'archive ouverte pluridisciplinaire HAL, est destinée au dépôt et à la diffusion de documents scientifiques de niveau recherche, publiés ou non, émanant des établissements d'enseignement et de recherche français ou étrangers, des laboratoires publics ou privés. 


\title{
Displacement dependent pressure load for finite deflection of doubly-curved thick shells and plates
}

\author{
Marco Amabili, Ivan D. Breslavsky \\ Department of Mechanical Engineering, McGill University, 817 Sherbrooke Street West, Montreal, Canada H3A 0C3
}

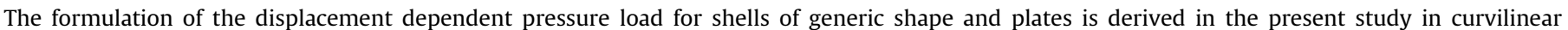

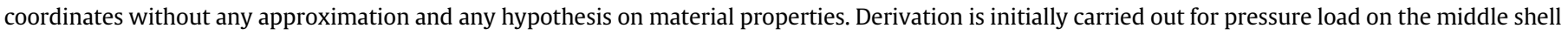

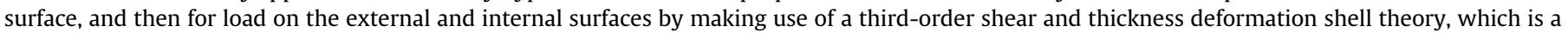

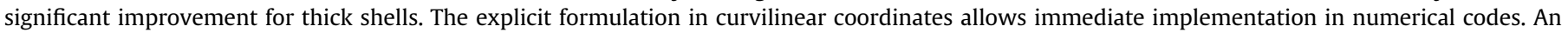

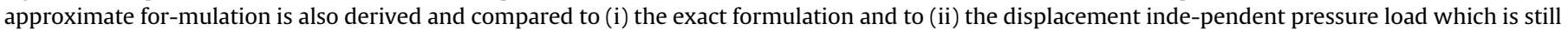

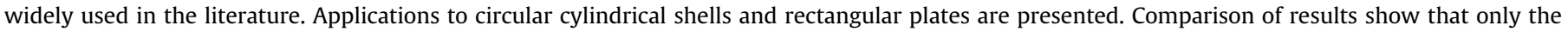

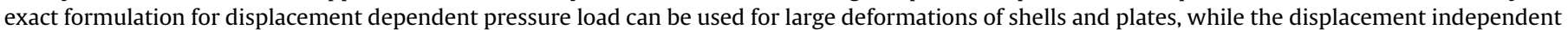
pressure load can be used only in case of small displacements of the middle surface (or middle plane).

Pressure, Shells, Plates, Displacement dependent pressure Large deformations

\section{Introduction}

Pressure is commonly used to represent load by pressurized gas, liquid and wind just to cite a few cases. By definition it is displacement dependent in the sense that this load changes with the deformation of the structures. In fact, pressure is orthogonal to the surface on which it is applied, so its local direction changes with the deformation of the surface. At the same time the surface is stretched or shrunk, so the resulting area on which the pressure is applied changes.

Displacement dependent pressure complicates the analysis, so it is sometimes replaced with constant direction distributed load in Lagrangian description (strains and stresses evaluated in the original undeformed configuration), especially in analytical studies. This approximation can lead to different type of accuracy, due mainly to the large or small deformation of the structure. In particular, for shells and plates made of soft materials (linear elastic or hyperelastic materials, like rubbers or biomaterials), displacement dependent pressure load must be used since they usually undergo very large deformations.
The literature approaching the problem of displacement dependent loads is not that vast, considering the importance of the problem. Almost all studies deal with finite element formulation and they use tensor notation and introduce at some point approximations. Koiter [1] derived the virtual work done by pressure for closed thin shells by using the principle that this is given by the product of the external pressure and the volume variation of the region of space enclosed by the middle surface of the shell. He kept the quadratic and cubic geometrically nonlinear terms in the calculation of the volume variation. The same approach was followed by Libai and Simmonds [2] that applied the formulation to closed shells and membranes, specifying the formulation for toroidal shells.

A general investigation of the mathematical properties of configuration-dependent loading is described by Sewell [3]. This study is not applied to shells and plates or uses shell theories.

Mang [4] derived the displacement dependent pressure stiffness matrices for shells in approximated way for finite element implementation. Argyris and Symeonidis [5] approached the problem of nonconservative (follower) forces by finite element formulation. In particular, the load correction stiffness matrix that represents the nonsymmetric contribution of the configurationdependent nonconservative loading to the tangent stiffness matrix of the element was derived. Chang and Sawamiphakdi [6] were able to study load due to follower forces by using a finite element incremental scheme. Schweizerhof and Ramm [7] derived the expression of the virtual work done by displacement dependent 
pressure for finite element incremental loading algorithm. The solution is obtained by incremental decomposition omitting nonlinear terms in the displacement increment.

Cheung and Zhu [8], while studying the postbuckling analysis of circular cylindrical shells under external pressure, derived the exact expression of the external virtual work done by displacement dependent pressure for finite element formulation. They expressed their formulation also in curvilinear coordinates for circular cylindrical shells, but not for shells with variable curvature. Zhu and Cheung [9] extended their study to postbuckling analysis of circular cylindrical shells under combined load, reporting the same derivation of the external virtual work done by displacement dependent pressure in curvilinear coordinates. The final expression (13) in [9] differs in one term with respect to their previous formulation, probably due to a typing error in Eq. (43) in [8].

Even in recent years most of the literature that is not based on finite element formulation still uses displacement independent pressure load, see e.g. [10]. Even if derivations for displacement dependent pressure load for shells are present in the literature, it seems that an exact formulation for doubly curved shells is not present as well as for thick shells with pressure applied on the internal or external surface.

The formulation of the displacement dependent pressure load for shells of generic shape is derived in the present study in curvilinear coordinates without any approximation and any hypothesis on material properties (i.e. it is valid also for hyperplastic materials). This allows immediate implementation in numerical codes. The derivation, not being based on the variation of the enclosed volume, is valid also for open shells and plates. A formulation for pressure applied on the external or internal shell surface instead of the middle plane is also obtained, which is a significant improvement for thick shells. An approximate expression is also derived and compared to (i) the exact formulation and to (ii) the displacement independent pressure load which is still widely used in the literature. Applications to circular cylindrical shells and rectangular plates are presented. Comparison of results show that only the exact formulation for displacement dependent pressure load can be used for large deformations of shells and plates, while the displacement independent pressure load can be used only in case of small displacements of the middle surface (or middle plane).

\section{Virtual work by displacement dependent pressure}

Pressure is a specific type of load that depends on the deformation of the shell in Lagrangian description. Here for simplicity the pressure is assumed to be applied to the middle surface of the shell. In the next section, the pressure is considered applied to the external or internal shell surface.

A shell of arbitrary shape and material is considered; the theory is valid for shells made of isotropic, laminated composite or functionally graded materials, as well as hyperelastic materials. The shell principal curvilinear coordinates are $\left(\alpha_{1}, \alpha_{2}\right)$. The displacements of an arbitrary point of coordinates $\left(\alpha_{1}, \alpha_{2}\right)$ taken on the middle surface of the shell are denoted by $u, v$ and $w$, in the $\alpha_{1}$, $\alpha_{2}$ and $z$ directions, respectively; these displacements do not have to be small. The normal displacement $w$ is taken positive outward from the center of the smallest radius of curvature as shown in Fig. 1. In the figure $R_{1}$ and $R_{2}$ (functions of the coordinates $\alpha_{1}$ and $\alpha_{2}$ ) are the principal radii of curvature in $\alpha_{1}$ and $\alpha_{2}$ directions, respectively.

Initial geometric imperfections of the shell associated with zero initial tension are denoted by displacement $w_{0}$ in $z$ direction, also taken positive outward and measured from the ideal middle

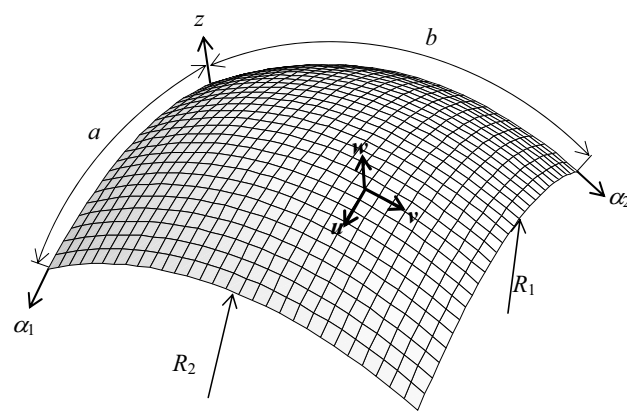

Fig. 1. Displacements of the middle surface of the shell and coordinate system.

surface. Imperfections other than in normal directions are not considered and an initial stress-free state is assumed.

The thickness $h$ of the shell is assumed to be small compared to the principal radii of curvature of the shell, but not very small, so that moderately thick shells can be considered with accuracy.

Using the curvilinear coordinates, the middle surface of the shell can be described by the vector equation

$\mathbf{r}=\mathbf{r}\left(\alpha_{1}, \alpha_{2}\right)$

After deformation, the middle surface is given by [11]

$\mathbf{r}^{\prime}=\mathbf{r}^{\prime}\left(\alpha_{1}, \alpha_{2}\right)=\mathbf{r}\left(\alpha_{1}, \alpha_{2}\right)+u \mathbf{e}_{1}+v \mathbf{e}_{2}+w \mathbf{e}_{n}$,

where $\mathbf{e}_{1}, \mathbf{e}_{2}, \mathbf{e}_{n}$ are the three unit vectors of the undeformed shell surface in directions $\alpha_{1}, \alpha_{2}$ and $z$, respectively. The three unit vectors of the undeformed surface are defined as [11,12]

$\mathbf{e}_{1}=\frac{1}{A_{1}} \frac{\partial \mathbf{r}}{\partial \alpha_{1}}, \quad \mathbf{e}_{2}=\frac{1}{A_{2}} \frac{\partial \mathbf{r}}{\partial \alpha_{2}}, \quad \mathbf{e}_{n}=\mathbf{e}_{1} \wedge \mathbf{e}_{2}$

where $A_{1}$ and $A_{2}$ are the Lamé parameters. Similarly, after deformation, the unit vectors become

$\mathbf{e}_{1}^{\prime}=\frac{1}{A_{1}^{\prime}} \frac{\partial \mathbf{r}^{\prime}}{\partial \alpha_{1}}$

$\mathbf{e}_{2}^{\prime}=\frac{1}{A_{2}^{\prime}} \frac{\partial \mathbf{r}^{\prime}}{\partial \alpha_{2}}$

$\mathbf{e}_{n}^{\prime}=\mathbf{e}_{1}^{\prime} \wedge \mathbf{e}_{2}^{\prime}$

where $A_{1}^{\prime}$ and $A_{2}^{\prime}$ are the Lamé parameters after shell deformation. The following two relationships follow [11]

$\frac{1}{A_{1}} \frac{\partial \mathbf{r}^{\prime}}{\partial \alpha_{1}}=\left(1+\bar{\varepsilon}_{1}\right) \mathbf{e}_{1}+\bar{\omega}_{1} \mathbf{e}_{2}-\bar{\Theta} \mathbf{e}_{n}$

$\frac{1}{A_{2}} \frac{\partial \mathbf{r}^{\prime}}{\partial \alpha_{2}}=\bar{\omega}_{2} \mathbf{e}_{1}+\left(1+\bar{\varepsilon}_{2}\right) \mathbf{e}_{2}-\bar{\Psi} \mathbf{e}_{n}$

where $\bar{\varepsilon}_{1}, \bar{\varepsilon}_{2}, \bar{\omega}_{1}, \bar{\omega}_{2}, \bar{\Theta}$, and $\bar{\Psi}$ are defined by [11]

$\bar{\varepsilon}_{1}=\left(\frac{1}{A_{1}} \frac{\partial u}{\partial \alpha_{1}}+\frac{1}{A_{1} A_{2}} \frac{\partial A_{1}}{\partial \alpha_{2}} v+\frac{w}{R_{1}}\right)$,

$\bar{\varepsilon}_{2}=\left(\frac{1}{A_{2}} \frac{\partial v}{\partial \alpha_{2}}+\frac{1}{A_{1} A_{2}} \frac{\partial A_{2}}{\partial \alpha_{1}} u+\frac{w}{R_{2}}\right)$,

$\bar{\omega}_{1}=\left(\frac{1}{A_{1}} \frac{\partial v}{\partial \alpha_{1}}-\frac{1}{A_{1} A_{2}} \frac{\partial A_{1}}{\partial \alpha_{2}} u\right)$

$\bar{\omega}_{2}=\left(\frac{1}{A_{2}} \frac{\partial u}{\partial \alpha_{2}}-\frac{1}{A_{1} A_{2}} \frac{\partial A_{2}}{\partial \alpha_{1}} v\right)$,

$\bar{\Theta}=\left(-\frac{1}{A_{1}} \frac{\partial w}{\partial \alpha_{1}}+\frac{u}{R_{1}}\right)$,

$\bar{\Psi}=\left(-\frac{1}{A_{2}} \frac{\partial w}{\partial \alpha_{2}}+\frac{v}{R_{2}}\right)$. 
The Lamé parameters after deformation are calculated as

$$
A_{1}^{\prime}=A_{1} \sqrt{\left(1+\bar{\varepsilon}_{1}\right)^{2}+\bar{\omega}_{1}^{2}+\bar{\Theta}^{2}}
$$

$$
A_{2}^{\prime}=A_{1} \sqrt{\left(1+\bar{\varepsilon}_{2}\right)^{2}+\bar{\omega}_{2}^{2}+\bar{\Psi}^{2}}
$$

The unit vectors of the deformed surface are directly related to those of the original surface

$\mathbf{e}_{1}^{\prime}=\frac{A_{1}}{A_{1}^{\prime}}\left[\left(1+\bar{\varepsilon}_{1}\right) \mathbf{e}_{1}+\bar{\omega}_{1} \mathbf{e}_{2}-\bar{\Theta} \mathbf{e}_{n}\right]$,

$\mathbf{e}_{2}^{\prime}=\frac{A_{2}}{A_{2}^{\prime}}\left[\bar{\omega}_{2} \mathbf{e}_{1}+\left(1+\bar{\varepsilon}_{2}\right) \mathbf{e}_{2}-\bar{\Psi} \mathbf{e}_{n}\right]$.

Using the expressions (8) and (9), the following equation is obtained

$$
\begin{aligned}
\mathbf{e}_{n}^{\prime}= & \mathbf{e}_{1}^{\prime} \wedge \mathbf{e}_{2}^{\prime}=\frac{A_{1} A_{2}}{A_{1}^{\prime} A_{2}^{\prime}}\left\{\left[\left(1+\bar{\varepsilon}_{1}\right)\left(1+\bar{\varepsilon}_{2}\right)-\bar{\omega}_{1} \bar{\omega}_{2}\right] \mathbf{e}_{n}+\left[\left(1+\bar{\varepsilon}_{2}\right) \bar{\Theta}-\bar{\omega}_{1} \bar{\Psi}\right] \mathbf{e}_{1}\right. \\
& \left.+\left[\left(1+\bar{\varepsilon}_{1}\right) \bar{\Psi}-\bar{\omega}_{2} \bar{\Theta}\right] \mathbf{e}_{2}\right\} .
\end{aligned}
$$

The virtual work done by pressure becomes

$$
\begin{aligned}
W= & \iint_{\Omega} p\left(u \mathbf{e}_{1}+v \mathbf{e}_{2}+w \mathbf{e}_{n}\right) \cdot \mathbf{e}_{n}^{\prime} A_{1}^{\prime} A_{2}^{\prime} \mathrm{d} \alpha_{1} \mathrm{~d} \alpha_{2} \\
= & \iint_{\Omega} p\left\{w\left[\left(1+\bar{\epsilon}_{1}\right)\left(1+\bar{\epsilon}_{2}\right)-\bar{\omega}_{1} \bar{\omega}_{2}\right]+u\left[\left(1+\bar{\epsilon}_{2}\right) \bar{\Theta}-\bar{\omega}_{1} \bar{\Psi}\right]\right. \\
& \left.+v\left[\left(1+\bar{\epsilon}_{1}\right) \bar{\Psi}-\bar{\omega}_{2} \bar{\Theta}\right]\right\} A_{1} A_{2} \mathrm{~d} \alpha_{1} \mathrm{~d} \alpha_{2},
\end{aligned}
$$

where $\Omega$ is the undeformed middle surface of the shell. Eliminating higher-order terms in Eq. (11) gives the following approximate expression

$$
W \simeq \iint_{\Omega} p\left[w\left(1+\bar{\epsilon}_{1}+\bar{\epsilon}_{2}\right)+u \bar{\Theta}+v \bar{\Psi}\right] A_{1} A_{2} \mathrm{~d} \alpha_{1} \mathrm{~d} \alpha_{2} .
$$

In the numerical result section, calculations by using Eqs. (11) and (12) are presented for shells and plates, and compared to results obtained keeping only the linear term $p w$ in these equations.

Eq. (11) specified for a circular cylindrical shell gives

$$
\begin{aligned}
W= & \int_{0}^{L} \int_{0}^{2 \pi} p\left\{w\left[\left(1+\frac{\partial u}{\partial x}\right)\left(1+\frac{\partial v}{R \partial \theta}+\frac{w}{R}\right)-\frac{\partial v}{\partial x} \frac{\partial u}{R \partial \theta}\right]\right. \\
& +u\left[-\left(1+\frac{\partial v}{R \partial \theta}+\frac{w}{R}\right) \frac{\partial w}{\partial x}-\frac{\partial v}{\partial x}\left(-\frac{\partial w}{R \partial \theta}+\frac{v}{R}\right)\right] \\
& \left.+v\left[\left(1+\frac{\partial u}{\partial x}\right)\left(-\frac{\partial w}{R \partial \theta}+\frac{v}{R}\right)+\frac{\partial u}{R \partial \theta} \frac{\partial w}{\partial x}\right]\right\} \mathrm{d} x R \mathrm{~d} \theta .
\end{aligned}
$$

Eq. (13) is analogous to the expression obtained for the external virtual work done by displacement dependent pressure in reference [9], while there is a difference in the last term in square parenthesis with respect to reference [8], that seems therefore incorrect (probably there is a typing error in Eq. (44) in [8]).

Eq. (12) specified for a circular cylindrical shell gives

$W \simeq p \int_{0}^{L} \int_{0}^{2 \pi}\left[w\left(1+\frac{\partial u}{\partial x}+\frac{\partial v}{R \partial \theta}+\frac{w}{R}\right)-u \frac{\partial w}{\partial x}-v \frac{\partial w}{R \partial \theta}\right] \mathrm{d} x R \mathrm{~d} \theta$

Eq. (11) specified for a rectangular plate gives

$$
\begin{aligned}
W= & \int_{0}^{a} \int_{0}^{b} p\left[w\left(1+\frac{\partial u}{\partial x}+\frac{\partial v}{\partial y}+\frac{\partial u}{\partial x} \frac{\partial v}{\partial y}-\frac{\partial v}{\partial x} \frac{\partial u}{\partial y}\right)\right. \\
& \left.+u\left(-\frac{\partial w}{\partial x}-\frac{\partial w}{\partial x} \frac{\partial v}{\partial y}+\frac{\partial w}{\partial y} \frac{\partial v}{\partial x}\right)+v\left(-\frac{\partial w}{\partial y}-\frac{\partial w}{\partial y} \frac{\partial u}{\partial x}+\frac{\partial w}{\partial x} \frac{\partial u}{\partial y}\right)\right] \mathrm{d} x \mathrm{~d} y
\end{aligned}
$$

Eq. (12) specified for a rectangular plate shell gives

$W \simeq p \int_{0}^{a} \int_{0}^{b}\left[w\left(1+\frac{\partial u}{\partial x}+\frac{\partial v}{\partial y}\right)-u \frac{\partial w}{\partial x}-v \frac{\partial w}{\partial y}\right] \mathrm{d} x \mathrm{dy}$

\section{Pressure applied to the shell surface}

In this section, the simplification of applying the displacement dependent pressure to the shell middle surface is removed and the pressure is directly applied to the external or internal shell surface.

A shell theory that retains shear and thickness deformation and rotary inertia is used. The literature review on shell theories with thickness deformation is not wide. An accurate linear shell theory that takes into account thickness stretching has been developed by Carrera et al. [13] and Ferreira et al. [14]. Büchter et al. [15] and Bischoff and Ramm [16,17] elaborated seven-parameter shell theories that introduce a linear and a quadratic term to describe the stretching of the shell thickness. Also Parisch [18], Sansour [19] and Sansour et al. [20] have been developed independently shell theories that introduce quadratic assumption of the transverse shell displacement over the shell thickness for finite element implementation. A first-order shear deformation shell theory with seven parameters and exact nonlinear deformations, under the framework of the Lagrangian description, has been derived in tensor notation by Arciniega and Reddy [21,22] and Payette and Reddy [23] for laminated and functionally graded shells. Amabili [24] introduced a first-order thickness stretching theory with higher-order shear deformation that uses 6 independent parameters. Amabili [25] introduced a geometrically non-linear shell theory allowing for third-order thickness stretching, higher-order shear deformation and rotary inertia by using eight parameters. Alijani and Amabili [26] introduced a second-order thickness stretching theory that uses 7 parameters to describe the deformation of plates and, possibly for the first time, retains full nonlinear terms for displacements, rotations and thickness deformation. Finally Amabili [27] introduced the full nonlinear terms in the 8 parameter theory for shells.

Using the curvilinear coordinates, the shell surface at distance $z$ from the middle surface can be described by the vector equation

$\mathbf{r}=\mathbf{r}\left(\alpha_{1}, \alpha_{2}, z\right)$

The position of a point of the shell at distance $z$ from the middle surface is given, after deformation, by

$\mathbf{r}^{\prime}=\mathbf{r}^{\prime}\left(\alpha_{1}, \alpha_{2}, z\right)=\mathbf{r}\left(\alpha_{1}, \alpha_{2}, z\right)+u_{1} \mathbf{e}_{1}+u_{2} \mathbf{e}_{2}+u_{3} \mathbf{e}_{n}$,

where $\mathbf{e}_{1}, \mathbf{e}_{2}, \mathbf{e}_{n}$ are the three unit vectors of the undeformed shell surface at distance $z$ from the middle surface in directions $\alpha_{1}, \alpha_{2}$ and $z$, respectively and $u_{1}, u_{2}$, and $u_{3}$ are the displacements of a point (at distance $z$ from the middle shell surface) in $\alpha_{1}, \alpha_{2}$ and $z$ directions, respectively, which are given by [27]

$u_{1}=\left[1+\left(z / R_{1}\right)\right] u+z \phi_{1}+z^{2} \psi_{1}+z^{3} \gamma_{1}$,

$u_{2}=\left[1+\left(z / R_{2}\right)\right] v+z \phi_{2}+z^{2} \psi_{2}+z^{3} \gamma_{2}$,

$u_{3}=w+w_{0}+z \chi_{1}+z^{2} \chi_{2}+z^{3} \chi_{3}$

$R_{1}$ and $R_{2}$ are the principal radii of curvature referred to the middle surface of the shell. In Eq. (19a)-(19c)u, $v$ and $w$ are the displacements of a point on the shell middle surface in $\alpha_{1}, \alpha_{2}$ and $z$ directions, respectively, $\phi_{1}$ and $\phi_{2}$ are the rotations of the transverse normal at $z=0$ (i.e. referred to the middle surface) due to bending (but not shear deformation) about the $\alpha_{2}$ and $\alpha_{1}$ axes, respectively, and $\chi_{1}, \chi_{2}$ and $\chi_{3}$ are three parameters linked to the thickness deformation per unit thickness. Then $\psi_{1}, \psi_{2}, \gamma_{1}$ and $\gamma_{2}$, are functions to be determined in terms of $u, v, w, \phi_{1}, \phi_{2}, \chi_{1}, \chi_{2}$ and $\chi_{3}$ which are the eight parameters describing the shell deformation. By using the condition of vanishing of the shear stresses $\tau_{13}$ and $\tau_{23}$ at the top and the bottom surfaces of the shell, 
the following expressions are obtained [27]:

$\psi_{1}=\frac{1}{3 R_{1}} \phi_{1}+\frac{1}{3 R_{1}} \frac{\partial\left(w+w_{0}\right)}{A_{1} \partial \alpha_{1}}-\frac{1}{2} \frac{\partial \chi_{1}}{A_{1} \partial \alpha_{1}}+\frac{h^{2}}{12 R_{1}} \frac{\partial \chi_{2}}{A_{1} \partial \alpha_{1}}-\frac{h^{2}}{8} \frac{\partial \chi_{3}}{A_{1} \partial \alpha_{1}}$

$\gamma_{1}=-\frac{4}{3 h^{2}}\left(\frac{\partial\left(w+w_{0}\right)}{A_{1} \partial \alpha_{1}}+\phi_{1}\right)+\frac{1}{2 R_{1}} \frac{\partial \chi_{1}}{A_{1} \partial \alpha_{1}}-\frac{1}{3} \frac{\partial \chi_{2}}{A_{1} \partial \alpha_{1}}+\frac{h^{2}}{24 R_{1}} \frac{\partial \chi_{3}}{A_{1} \partial \alpha_{1}}$,

$\psi_{2}=\frac{1}{3 R_{2}} \phi_{2}+\frac{1}{3 R_{2}} \frac{\partial\left(w+w_{0}\right)}{A_{2} \partial \alpha_{2}}-\frac{1}{2} \frac{\partial \chi_{1}}{A_{2} \partial \alpha_{2}}+\frac{h^{2}}{12 R_{2}} \frac{\partial \chi_{2}}{A_{2} \partial \alpha_{2}}-\frac{h^{2}}{8} \frac{\partial \chi_{3}}{A_{2} \partial \alpha_{2}}$,

$\gamma_{2}=-\frac{4}{3 h^{2}}\left(\frac{\partial\left(w+w_{0}\right)}{A_{2} \partial \alpha_{2}}+\phi_{2}\right)+\frac{1}{2 R_{2}} \frac{\partial \chi_{1}}{A_{2} \partial \alpha_{2}}-\frac{1}{3} \frac{\partial \chi_{2}}{A_{2} \partial \alpha_{2}}+\frac{h^{2}}{24 R_{2}} \frac{\partial \chi_{3}}{A_{2} \partial \alpha_{2}}$

where the Lamé parameters $A_{1}$ and $A_{2}$ are referred to the middle surface of the shell. Eqs. (19) and (20) are for a 3rd order shear deformation theory of shells with 3rd order thickness deformation. They can be reduced to simpler shell theories setting to zero some of the 8 parameters (e.g. setting to zero the 3 parameters $\chi_{1}$, $\chi_{2}$ and $\chi_{3}$ related to the thickness deformation and reducing to just 3 rd order shear deformation theory). In case of classical Novozhilov shell theory, it is necessary to set $\psi_{1}=\psi_{2}=\gamma_{1}=\gamma_{2}=$ $\chi_{1}=\chi_{2}=\chi_{3}=0$ and, after neglecting nonlinear terms, to set

$\varphi_{1} \simeq-\frac{\partial w}{A_{1} \partial \alpha_{1}}, \quad \varphi_{2} \simeq-\frac{\partial w}{A_{2} \partial \alpha_{2}}$,

The three unit vectors of the undeformed surface at distance $z$ from the middle surface are defined as [11,12]

$\mathbf{e}_{1}=\frac{1}{A_{1}(z)} \frac{\partial \mathbf{r}}{\partial \alpha_{1}}$,

$\mathbf{e}_{2}=\frac{1}{A_{2}(z)} \frac{\partial \mathbf{r}}{\partial \alpha_{2}}$

$\mathbf{e}_{n}=\mathbf{e}_{1} \wedge \mathbf{e}_{2}$,

where

$A_{1}(z)=A_{1}\left(1+z / R_{1}\right)$

$A_{2}(z)=A_{2}\left(1+z / R_{2}\right)$,

are the Lamé parameters of the surface at distance $z$.

Similarly, after deformation, the unit vectors become

$\mathbf{e}_{1}^{\prime}=\frac{1}{A_{1}^{\prime}(z)} \frac{\partial \mathbf{r}^{\prime}}{\partial \alpha_{1}}$,

$\mathbf{e}_{2}^{\prime}=\frac{1}{A_{2}^{\prime}(z)} \frac{\partial \mathbf{r}^{\prime}}{\partial \alpha_{2}}$

$\mathbf{e}_{n}^{\prime}=\mathbf{e}_{1}^{\prime} \wedge \mathbf{e}_{2}^{\prime}$,

where $A_{1}^{\prime}(z)$ and $A_{2}^{\prime}(z)$ are the Lamé parameters of the surface at distance $z$ after shell deformation. The following two relationships follow [11]

$\frac{1}{A_{1}(z)} \frac{\partial^{\prime}}{\partial \alpha_{1}}=\left(1+\varepsilon_{1}\right) \mathbf{e}_{1}+\omega_{1} \mathbf{e}_{2}-\Theta \mathbf{e}_{n}$,

$\frac{1}{A_{2}(z)} \frac{\partial \mathbf{r}^{\prime}}{\partial \alpha_{2}}=\omega_{2} \mathbf{e}_{1}+\left(1+\varepsilon_{2}\right) \mathbf{e}_{2}-\Psi \mathbf{e}_{n}$,

where $\varepsilon_{1}, \varepsilon_{2}, \omega_{1}, \omega_{2}, \Theta$, and $\Psi$ are defined by [11,28]

$\varepsilon_{1}=\frac{1}{1+\left(z / R_{1}\right)}\left(\frac{1}{A_{1}} \frac{\partial u_{1}}{\partial \alpha_{1}}+\frac{1}{A_{1} A_{2}} \frac{\partial A_{1}}{\partial \alpha_{2}} u_{2}+\frac{u_{3}}{R_{1}}\right)$, $\varepsilon_{2}=\frac{1}{1+\left(z / R_{2}\right)}\left(\frac{1}{A_{2}} \frac{\partial u_{2}}{\partial \alpha_{2}}+\frac{1}{A_{1} A_{2}} \frac{\partial A_{2}}{\partial \alpha_{1}} u_{1}+\frac{u_{3}}{R_{2}}\right)$,

$\omega_{1}=\frac{1}{1+\left(z / R_{1}\right)}\left(\frac{1}{A_{1}} \frac{\partial u_{2}}{\partial \alpha_{1}}-\frac{1}{A_{1} A_{2}} \frac{\partial A_{1}}{\partial \alpha_{2}} u_{1}\right)$,

$\omega_{2}=\frac{1}{1+\left(z / R_{2}\right)}\left(\frac{1}{A_{2}} \frac{\partial u_{1}}{\partial \alpha_{2}}-\frac{1}{A_{1} A_{2}} \frac{\partial A_{2}}{\partial \alpha_{1}} u_{2}\right)$,

$\Theta=\frac{1}{1+\left(z / R_{1}\right)}\left(-\frac{1}{A_{1}} \frac{\partial u_{3}}{\partial \alpha_{1}}+\frac{u_{1}}{R_{1}}\right)$,

$\Psi=\frac{1}{1+\left(z / R_{2}\right)}\left(-\frac{1}{A_{2}} \frac{\partial u_{3}}{\partial \alpha_{2}}+\frac{u_{2}}{R_{2}}\right)$.

The Lamé parameters after deformation are calculated as

$A_{1}^{\prime}(z)=A_{1}(z) \sqrt{\left(1+\varepsilon_{1}\right)^{2}+\omega_{1}^{2}+\Theta^{2}}$,

$A_{2}^{\prime}(z)=A_{1}(z) \sqrt{\left(1+\varepsilon_{2}\right)^{2}+\omega_{2}^{2}+\Psi^{2}}$.

The unit vectors of the deformed surface are directly related to those of the original surface

$\mathbf{e}_{1}^{\prime}=\frac{A_{1}(z)}{A_{1}^{\prime}(z)}\left[\left(1+\varepsilon_{1}\right) \mathbf{e}_{1}+\omega_{1} \mathbf{e}_{2}-\Theta \mathbf{e}_{n}\right]$,

$\mathbf{e}_{2}^{\prime}=\frac{A_{2}(z)}{A_{2}^{\prime}(z)}\left[\omega_{2} \mathbf{e}_{1}+\left(1+\varepsilon_{2}\right) \mathbf{e}_{2}-\Psi \mathbf{e}_{n}\right]$.

Using the expressions (26) and (27), the following equation is obtained

$$
\begin{aligned}
\mathbf{e}_{n}^{\prime}= & \mathbf{e}_{1}^{\prime} \wedge \mathbf{e}_{2}^{\prime}=\frac{A_{1}(z) A_{2}(z)}{A_{1}^{\prime}(z) A_{2}^{\prime}(z)}\left\{\left[\left(1+\varepsilon_{1}\right)\left(1+\varepsilon_{2}\right)-\omega_{1} \omega_{2}\right] \mathbf{e}_{n}\right. \\
& +\left[\left(1+\varepsilon_{2}\right) \Theta-\omega_{1} \Psi\right] \mathbf{e}_{1} \\
& \left.+\left[\left(1+\varepsilon_{1}\right) \Psi-\omega_{2} \Theta\right] \mathbf{e}_{2}\right\} .
\end{aligned}
$$

The virtual work done by pressure becomes

$$
\begin{aligned}
W= & {\left[\iint_{\Omega} p\left(u_{1} \mathbf{e}_{1}+u_{2} \mathbf{e}_{2}+u_{3} \mathbf{e}_{n}\right) \cdot \mathbf{e}_{n}^{\prime} A_{1}^{\prime}(z) A_{2}^{\prime}(z) d \alpha_{1} \mathrm{~d} \alpha_{2}\right]_{z= \pm h / 2} } \\
= & {\left[\int \int _ { \Omega } p \left\{u_{3}\left[\left(1+\varepsilon_{1}\right)\left(1+\varepsilon_{2}\right)-\omega_{1} \omega_{2}\right]+u_{1}\left[\left(1+\varepsilon_{2}\right) \Theta-\omega_{1} \Psi\right]\right.\right.} \\
& \left.\left.+u_{2}\left[\left(1+\varepsilon_{1}\right) \Psi-\omega_{2} \Theta\right]\right\} A_{1}\left(1+z / R_{1}\right) A_{2}\left(1+z / R_{2}\right) \mathrm{d} \alpha_{1} \mathrm{~d} \alpha_{2}\right]_{z= \pm h / 2},
\end{aligned}
$$

where $\Omega$ is the undeformed surface of the shell where the pressure is applied and $z=h / 2$ for external pressure and $z=-h / 2$ for internal pressure. In general, $p$ can be a time-dependent or constant pressure.

The following approximation can be introduced

$\frac{1}{1+(z / R)} \simeq 1-\frac{z}{R}+\frac{z^{2}}{R^{2}}-\frac{z^{3}}{R^{3}}$

Eq. (29) specified for a complete circular cylindrical shell of radius $R$ (measured on the middle surface), length $L$ and keeping only linear terms in the thickness $h$ (i.e. terms multiplied by $h^{2}, h^{3}$, $\ldots$ are neglected) gives

$$
\begin{aligned}
W= & \int_{0}^{L} \int_{0}^{2 \pi} p\left\{\left(w+z \chi_{1}\right)\left[\left(1+\frac{\partial u}{\partial x}\right)\left(1+\frac{\partial v}{R \partial \theta}+\frac{w}{R}\right)-\frac{\partial v}{\partial x} \frac{\partial u}{R \partial \theta}\right]\right. \\
& +\left(u+z \phi_{1}\right)\left[-\left(1+\frac{\partial v}{R \partial \theta}+\frac{w}{R}\right) \frac{\partial w}{\partial x}-\frac{\partial v}{\partial x}\left(-\frac{\partial w}{R \partial \theta}+\frac{v}{R}\right)\right] \\
& +\left(v+z \phi_{2}+z \frac{v}{R}\right)\left[\left(1+\frac{\partial u}{\partial x}\right)\left(-\frac{\partial w}{R \partial \theta}+\frac{v}{R}\right)+\frac{\partial u}{R \partial \theta} \frac{\partial w}{\partial x}\right] \\
& +z w\left[\left(1+\frac{\partial u}{\partial x}\right)\left(\frac{\partial \phi_{2}}{R \partial \theta}+\frac{\chi_{1}}{R}-\frac{w}{R^{2}}\right)+\left(1+\frac{\partial v}{R \partial \theta}+\frac{w}{R}\right) \frac{\partial \phi_{1}}{\partial x}\right. \\
& \left.-\frac{\partial v}{\partial x} \frac{\partial \phi_{1}}{R}-\frac{\partial u}{R \partial \theta} \frac{\partial \phi_{2}}{\partial x}\right]
\end{aligned}
$$




$$
\begin{aligned}
& +z u\left[-\left(1+\frac{\partial v}{R \partial \theta}+\frac{w}{R}\right) \frac{\partial \chi_{1}}{\partial x}-\frac{\partial w}{\partial x}\left(\frac{\partial \phi_{2}}{R \partial \theta}+\frac{\chi_{1}}{R}-\frac{w}{R^{2}}\right)\right. \\
& -\frac{\partial v}{\partial x}\left(\frac{\phi_{2}}{R}-\frac{\partial \chi_{1}}{R \partial \theta}+\frac{\partial w}{R^{2} \partial \theta}\right) \\
& \left.-\left(-\frac{\partial w}{R \partial \theta}+\frac{v}{R}\right)\left(\frac{\partial \phi_{2}}{\partial x}+\frac{\partial v}{R \partial x}\right)\right] \\
& +z v\left[\left(1+\frac{\partial u}{\partial x}\right)\left(\frac{\phi_{2}}{R}-\frac{\partial \chi_{1}}{R \partial \theta}+\frac{\partial w}{R^{2} \partial \theta}\right)+\left(-\frac{\partial w}{R \partial \theta}+\frac{v}{R}\right) \frac{\partial \phi_{1}}{\partial x}\right. \\
& \left.\left.+\frac{\partial u}{R \partial \theta} \frac{\partial \chi_{1}}{\partial x}+\frac{\partial w}{\partial x}\left(\frac{\partial \phi_{1}}{R \partial \theta}-\frac{\partial u}{R^{2} \partial \theta}\right)\right]\right\}_{z= \pm h / 2}^{\mathrm{d} x\left(R \pm \frac{h}{2}\right) \mathrm{d} \theta}
\end{aligned}
$$

In case of classical Novozhilov shell theory, Eq. (31) reduces to

$$
\begin{aligned}
W= & \int_{0}^{L} \int_{0}^{2 \pi} p\left\{w\left[\left(1+\frac{\partial u}{\partial x}\right)\left(1+\frac{\partial v}{R \partial \theta}+\frac{w}{R}\right)-\frac{\partial v}{\partial x} \frac{\partial u}{R \partial \theta}\right]\right. \\
& +\left(u-z \frac{\partial w}{\partial x}\right)\left[-\left(1+\frac{\partial v}{R \partial \theta}+\frac{w}{R}\right) \frac{\partial w}{\partial x}-\frac{\partial v}{\partial x}\left(-\frac{\partial w}{R \partial \theta}+\frac{v}{R}\right)\right] \\
& +\left(v-z \frac{\partial w}{R \partial \theta}+z \frac{v}{R}\right)\left[\left(1+\frac{\partial u}{\partial x}\right)\left(-\frac{\partial w}{R \partial \theta}+\frac{v}{R}\right)+\frac{\partial u}{R \partial \theta} \frac{\partial w}{\partial x}\right] \\
& +z w\left[\left(1+\frac{\partial u}{\partial x}\right)\left(-\frac{\partial^{2} w}{R^{2}}-\frac{w}{\theta^{2}}-\frac{w}{R^{2}}\right)\right. \\
& \left.-\left(1+\frac{\partial v}{R \partial \theta}+\frac{w}{R}\right) \frac{\partial^{2} w}{\partial x^{2}}+\frac{\partial v}{\partial x} \frac{\partial^{2} w}{R^{2}}+\frac{\partial \theta^{2}}{R} \partial \theta \frac{\partial^{2} w}{\partial x R \partial \theta}\right] \\
& +z u\left[-\frac{\partial w}{\partial x}\left(-\frac{\partial^{2} w}{R^{2} \partial \theta^{2}}-\frac{w}{R^{2}}\right)-\left(-\frac{\partial w}{R \partial \theta}+\frac{v}{R}\right)\left(-\frac{\partial^{2} w}{\partial x R \partial \theta}+\frac{\partial v}{R \partial x}\right)\right] \\
& \left.+z v\left[\left(\frac{\partial w}{R \partial \theta}-\frac{v}{R}\right) \frac{\partial^{2} w}{\partial x^{2}}+\frac{\partial w}{\partial x}\left(-\frac{\partial^{2} w}{\partial x R \partial \theta}-\frac{\partial u}{R^{2} \partial \theta}\right)\right]\right\}_{z= \pm h / 2}
\end{aligned}
$$$$
\mathrm{d} x\left(R \pm \frac{h}{2}\right) \mathrm{d} \theta
$$

Eq. (13) is obtained if $h$ is neglected in Eqs. (31) and (32).

Eq. (29) specified for a rectangular plate of in-plane dimensions $a, b$, and keeping only linear terms in the thickness $h$ gives

$$
\begin{aligned}
W= & \int_{0}^{a} \int_{0}^{b} p\left\{\left(w+z \chi_{1}\right)\left[\left(1+\frac{\partial u}{\partial x}\right)\left(1+\frac{\partial v}{\partial y}\right)-\frac{\partial v}{\partial x} \frac{\partial u}{\partial y}\right]\right. \\
& +\left(u+z \varphi_{1}\right)\left[-\left(1+\frac{\partial v}{\partial y}\right) \frac{\partial w}{\partial x}+\frac{\partial v}{\partial x} \frac{\partial w}{\partial y}\right] \\
& +\left(v+z \varphi_{2}\right)\left[-\left(1+\frac{\partial u}{\partial x}\right) \frac{\partial w}{\partial y}+\frac{\partial u}{\partial y} \frac{\partial w}{\partial x}\right] \\
& +z w\left[\left(1+\frac{\partial u}{\partial x}\right) \frac{\partial \varphi_{2}}{\partial y}+\left(1+\frac{\partial v}{\partial y}\right) \frac{\partial \varphi_{1}}{\partial x}-\frac{\partial v}{\partial x} \frac{\partial \varphi_{1}}{\partial y}-\frac{\partial u}{\partial y} \frac{\partial \varphi_{2}}{\partial x}\right] \\
& +z u\left[-\left(1+\frac{\partial v}{\partial y}\right) \frac{\partial \chi_{1}}{\partial x}-\frac{\partial w}{\partial x} \frac{\partial \varphi_{2}}{\partial y}+\frac{\partial v}{\partial x} \frac{\partial \chi_{1}}{\partial y}+\frac{\partial w}{\partial y} \frac{\partial \varphi_{2}}{\partial x}\right] \\
& \left.+z v\left[-\left(1+\frac{\partial u}{\partial x}\right) \frac{\partial \chi_{1}}{\partial y}-\frac{\partial w}{\partial y} \frac{\partial \varphi_{1}}{\partial x}+\frac{\partial u}{\partial y} \frac{\partial \chi_{1}}{\partial x}+\frac{\partial w}{\partial x} \frac{\partial \varphi_{1}}{\partial y}\right]\right\}_{z= \pm h / 2}^{\mathrm{d} x \mathrm{~d} y .}
\end{aligned}
$$

In case of classical Novozhilov shell theory, Eq. (33) reduces to

$$
\begin{aligned}
W= & \int_{0}^{a} \int_{0}^{b} p\left\{w\left[\left(1+\frac{\partial u}{\partial x}\right)\left(1+\frac{\partial v}{\partial y}\right)-\frac{\partial v}{\partial x} \frac{\partial u}{\partial y}\right]\right. \\
& +\left(u-z \frac{\partial w}{\partial x}\right)\left[-\left(1+\frac{\partial v}{\partial y}\right) \frac{\partial w}{\partial x}+\frac{\partial v}{\partial x} \frac{\partial w}{\partial y}\right] \\
& +\left(v-z \frac{\partial w}{\partial y}\right)\left[-\left(1+\frac{\partial u}{\partial x}\right) \frac{\partial w}{\partial y}+\frac{\partial u}{\partial y} \frac{\partial w}{\partial x}\right] \\
& +z w\left[-\left(1+\frac{\partial u}{\partial x}\right) \frac{\partial^{2} w}{\partial y^{2}}-\left(1+\frac{\partial v}{\partial y}\right) \frac{\partial^{2} w}{\partial x^{2}}+\left(\frac{\partial v}{\partial x}+\frac{\partial u}{\partial y}\right) \frac{\partial^{2} w}{\partial x \partial y}\right] \\
& \left.+z u\left[\frac{\partial w}{\partial x} \frac{\partial^{2} w}{\partial y^{2}}-\frac{\partial w}{\partial y} \frac{\partial^{2} w}{\partial x \partial y}\right]+z v\left[\frac{\partial w}{\partial y} \frac{\partial^{2} w}{\partial x^{2}}-\frac{\partial w}{\partial x} \frac{\partial^{2} w}{\partial x \partial y}\right]\right\}_{z= \pm h / 2}
\end{aligned}
$$

Eqs. (33) and (34) reduce to Eq. (15) if $h$ is neglected.

\section{Discretization and Lagrange Equations}

The displacements of the shell middle surface $u, v$ and $w$ are expanded by using the following expressions, which satisfy identically the geometric boundary conditions:

$u\left(\alpha_{1}, \alpha_{2}, t\right)=\sum_{m=1}^{M} \sum_{n=0}^{N} u_{m, n}(t) f_{1}\left(m, \alpha_{1}\right) g_{1}\left(n, \alpha_{2}\right)$,

$v\left(\alpha_{1}, \alpha_{2}, t\right)=\sum_{m=1}^{M} \sum_{n=0}^{N} v_{m, n}(t) f_{2}\left(m, \alpha_{1}\right) g_{2}\left(n, \alpha_{2}\right)$,

$w\left(\alpha_{1}, \alpha_{2}, t\right)=\sum_{m=1}^{M} \sum_{n=0}^{N} w_{m, n}(t) f_{3}\left(m, \alpha_{1}\right) g_{3}\left(n, \alpha_{2}\right)$,

where the variables have been separated $[28,29]$. In the case that a shell theory with shear and thickness deformation and rotary inertia is used, the following rotations of the transverse normal at $z=0$ (i.e. referred to the middle surface) due to bending (but not shear deformation) about the $\alpha_{2}$ and $\alpha_{1}$ axes, respectively, are also introduced

$\phi_{1}\left(\alpha_{1}, \alpha_{2}, t\right)=\sum_{m=1}^{M} \sum_{n=0}^{N} \phi_{1_{m, n}}(t) f_{4}\left(m, \alpha_{1}\right) g_{4}\left(n, \alpha_{2}\right)$,

$\phi_{2}\left(\alpha_{1}, \alpha_{2}, t\right)=\sum_{m=1}^{M} \sum_{n=0}^{N} \phi_{2_{m, n}}(t) f_{5}\left(m, \alpha_{1}\right) g_{5}\left(n, \alpha_{2}\right)$.

The following three expansions are used for the thickness deformation variables:

$\chi_{1}\left(\alpha_{1}, \alpha_{2}, t\right)=\sum_{m=1}^{M} \sum_{n=0}^{N} \chi_{1_{m, n}}(t) f_{6}\left(m, \alpha_{1}\right) g_{6}\left(n, \alpha_{2}\right)$,

$\chi_{2}\left(\alpha_{1}, \alpha_{2}, t\right)=\sum_{m=1}^{M} \sum_{n=0}^{N} \chi_{2_{m, n}}(t) f_{7}\left(m, \alpha_{1}\right) g_{7}\left(n, \alpha_{2}\right)$,

$\chi_{3}\left(\alpha_{1}, \alpha_{2}, t\right)=\sum_{m=1}^{M} \sum_{n=0}^{N} \chi_{3_{m, n}}(t) f_{8}\left(m, \alpha_{1}\right) g_{8}\left(n, \alpha_{2}\right)$.

For example, in case of a circular cylindrical shell that is simply supported at both ends and with zero thickness deformation at the ends, the geometric boundary conditions are $w=0, v=0, \phi_{2}=0$, $\chi_{1}=\chi_{2}=\chi_{3}=0$. The natural boundary conditions in that case are $N_{\alpha_{1}}=0, M_{\alpha_{1}}=0$ at the shell ends $\alpha_{1}=0, L$, where $N_{\alpha_{1}}$ is the axial stress resultant per unit length and $M_{\alpha_{1}}$ is the axial stress moment resultant per unit length.

The following vectorial notation is introduced:

$\mathbf{q}=\left\{u_{m, n} / h, v_{m, n} / h, w_{m, n} / h, \varphi_{1_{m, n}}, \varphi_{2_{m, n}}, \chi_{1_{m, n}}, \chi_{2_{m, n}} h, \chi_{3_{m, n}} h^{2}\right\}^{T}$,

$m=1, \ldots, M$ and $n=0, \ldots, N$

A non-dimensionalization of the time variables with respect to the shell thickness $h$ has been introduced in Eq. (36). The generic element (generalized coordinate) of the time-dependent vector $\mathrm{q}$ is referred to as $q_{j}$. The dimension of $\mathrm{q}$ is $\bar{N}$, which is the number of degrees of freedom (dofs) used in the mode expansion.

The generalized forces $Q_{j}$ are obtained by differentiation of the virtual work done by external forces [28]:

$Q_{j}=\frac{\partial W}{\partial q_{j}}$.

The Lagrange equations of motion are [28]

$\left.\frac{d}{d t} \frac{\partial T_{S}}{\partial q^{j}}\right)-\frac{\partial T_{S}}{\partial q_{j}}+\frac{\partial U_{S}}{\partial q_{j}}=Q_{j}, \quad j=1, \ldots, \bar{N}$

where $T_{S}$ and $U_{S}$ are the kinetic energy and potential elastic energy of the shell (see reference [28-30] for details), respectively, and 
$\partial T_{S} / \partial q_{j}=0 . U_{S}$ is derived taking care of geometric non-linearity and, eventually, of material non-linearity. The term derived from the maximum potential energy of the shell giving quadratic and cubic non-linearities, can be written in the form

$\frac{\partial U_{S}}{\partial q_{j}}=\sum_{i=1}^{\bar{N}} k_{j, i} q_{i}+\sum_{i, k=1}^{\bar{N}} s_{j, i, k} q_{i} q_{k}+\sum_{i, k, l=1}^{\bar{N}} r_{j, i, k, l} q_{i} q_{k} q_{l}, \quad j=1, \ldots, \bar{N}$

where the linear coefficients $k_{j, i}$, quadratic coefficients $s_{j, i, k}$ and cubic coefficients $r_{j, i, k, l}$ have long expressions that include also geometric imperfections.

Eq. (38) can be written in the following matrix form:

$$
M \ddot{\mathbf{q}}+C \dot{\mathbf{q}}+\left[\mathbf{K}+\mathbf{N}_{\mathbf{2}}(\mathbf{q})+\mathbf{N}_{\mathbf{3}}(\mathbf{q}, \mathbf{q})\right] \mathbf{q}=\mathbf{p}_{\mathbf{0}}(\mathbf{q}, t),
$$

where $\mathbf{M}$ is the non-diagonal mass matrix of dimension $\bar{N} \times \bar{N}$; $\mathbf{C}$ is the viscous damping matrix, which is added to the equations of motion to describe dissipation that is always present in dynamics (no dissipation is used for static problems); $\mathbf{K}$ is the linear stiffness matrix with elements $k_{j, i}, \mathbf{N}_{\mathbf{2}}$ gives the quadratic non-linear stiffness terms, $\mathbf{N}_{\mathbf{3}}$ denotes the cubic non-linear terms, and $\mathbf{p}_{\mathbf{0}}$ is the vector representing the displacement dependent pressure load, which are obtained by using Eqs. (11) and (37). The pressure load $\mathbf{p}_{\mathbf{0}}$, obtained by differentiating the virtual work $W$, is assumed in general to be also time dependant, which is the case in dynamics; in static problems the time dependence can be dropped. $\mathbf{p}_{\mathbf{0}}$ contains constant terms, linear and quadratic terms in the generalized coordinates.

In particular, by using Eq. (39), the generic elements $n_{2_{j, i}}$ and $n_{3_{j, i}}$ of the matrices $\mathbf{N}_{\mathbf{2}}$ and $\mathbf{N}_{\mathbf{3}}$, respectively, are given by

$n_{2_{j, i}}(\mathbf{q})=\sum_{k=1}^{N} S_{j, i, k} q_{k}$

$n_{3, i}(\mathbf{q}, \mathbf{q})=\sum_{k, l=1}^{\bar{N}} r_{j, i, k, l} q_{k} q_{l}$

In order to obtain the equations of motion in a suitable form for numerical implementation, the system Eq. (40) is multiplied by the inverse of mass matrix and then is written in the state-space form as follows

$$
\begin{aligned}
& \dot{\mathbf{q}}=\mathbf{y}, \\
& \dot{\mathbf{y}}=-\mathbf{M}^{-1} C \dot{\mathbf{q}}-\left[\mathbf{M}^{-1} K+\mathbf{M}^{-1} \mathbf{N}_{\mathbf{2}}(\mathbf{q})+\mathbf{M}^{-1} \mathbf{N}_{\mathbf{3}}(\mathbf{q}, \mathbf{q})\right] \mathbf{q}+\mathbf{M}^{-1} \mathbf{p}_{\mathbf{0}}(\mathbf{q}, t),
\end{aligned}
$$

where $\mathrm{y}$ is the vector of the generalized velocities. In particular, the dissipation term is given by

$\mathbf{M}^{-1} \mathbf{C}=\left[\begin{array}{ccc}2 \omega_{1} \zeta_{1} & \ldots & 0 \\ \vdots & \ddots & \vdots \\ 0 & \ldots & 2 \omega_{\bar{N}} \zeta_{\bar{N}}\end{array}\right]$

In Eq. (44) $\omega_{i}$ are the natural frequencies and $\zeta_{i}$ the corresponding modal damping ratios of each generalized coordinate. Matrix (44) is assumed to be diagonal in order to use modal damping.

\section{Numerical results}

Numerical results are obtained by solving Eq. (43) for large deflection with the computer program AUTO [31] for continuation and bifurcation analysis of ordinary differential equations and nonlinear algebraic equations starting from the trivial undeformed configuration. Calculations are performed for a circular cylindrical shell and a square plate made of isotropic and linearly elastic materials but in case of static large deformations.

\subsection{Circular cylindrical shell}

A simply supported circular cylindrical shell is investigated by using the classical geometrically nonlinear Novozhilov shell theory $[28,29,32]$ with the following boundary conditions: $w=0, v=0$, $N_{x}=0, M_{x}=0$, at $x=0, L$, where $N_{x}$ is the axial stress resultant per unit length and $M_{x}$ is the axial stress moment resultant per unit length. No geometric imperfections are included; shear and thickness deformations are neglected as well as rotary inertia. The dimensions and material properties are: radius $R=0.15 \mathrm{~m}$, length $L=0.52 \mathrm{~m}$, thickness $h=0.03 \mathrm{~m}$, Young's modulus $E=198 \times 10^{9} \mathrm{~Pa}$, Poisson ratio $\nu=0.3$ and mass density $\rho=7800 \mathrm{~kg} / \mathrm{m}^{3}$. The pressure is considered applied to the middle surface if not specified. The functions used in Eq. (35a)-(35c) for variable separation are $[28,29]$

$f_{1}\left(m, \alpha_{1}\right)=\cos \left(m \pi \alpha_{1} / L\right)$,

$f_{2}\left(m, \alpha_{1}\right)=f_{3}\left(m, \alpha_{1}\right)=\sin \left(m \pi \alpha_{1} / L\right)$,

$g_{1}\left(n, \alpha_{2}\right)=g_{3}\left(n, \alpha_{2}\right)=\cos \left(n \alpha_{2}\right)$,

$g_{2}\left(n, \alpha_{2}\right)=\sin \left(n \alpha_{2}\right)$,

with the in-plane axial and circumferential coordinates $0 \leq \alpha_{1} \leq L$ and $0 \leq \alpha_{2} \leq 2 \pi$, respectively. Introducing traditional notation, $\alpha_{1}=$ $x$ and $\alpha_{2}=\theta$. In expansion (35a-35c) only the following terms in the summatories are considered different from zero: $w_{1,0}, w_{3,0}, w_{5,0}, w_{7,0}$ $w_{9,0}, w_{11,0}, w_{1,2}, w_{3,2}, w_{5,2}, u_{1,0}, u_{3,0}, u_{5,0}, u_{7,0}, u_{9,0}, u_{11,0}, u_{1,2}, u_{3,2}, u_{5,2}$, $v_{1,2}, v_{3,2}, v_{5,2}, v_{1,4}, v_{3,4}, v_{5,4}$. The expansion used has $\bar{N}=24$ dofs. It allows to study with accuracy the axisymmetric deformation due to pressure load and postbuckling of the mode with 2 circumferential waves $(n=2)$; this is the first buckling mode for the studied shell. The shell theory and the approach have been validated in references $[28,29]$ for static and dynamic problems of thin shells, but without considering displacement dependent pressure.

The normalized (with respect to the thickness $h$ ) displacements of the shell versus pressure for different expressions of the pressure load are presented in Fig. 2. The exact formulation (13) is presented in continuous line and it is quite different from the solution obtained by displacement independent pressure (virtual work $W=p w$ ) for large deformation (since the shell is thick, a displacement of the order of the shell thickness $h$ is very large). The approximate solution (14) lies in between the other two in Fig. 2(a), which presents the radial displacement $w$ at mid-length, while it is close enough to the exact solution for the axial displacement $u$ at the edge $x=0$ in Fig. 2(b). It must be observed that Fig. 2 has been obtained removing the possibility to the shell to buckle in case of negative (i.e. external) pressure.

The case when buckling is allowed is presented in Fig. 3. A pitchfork bifurcation appears in Fig. 3(a) for negative pressures on the same branch of the solution presented in Fig. 2(a). A new branch of the static solution appears after the pitchfork bifurcation and corresponds to the post-buckling configuration of the shell. The buckling pressure (i.e. the pressure at which the pitchfork bifurcation appears) is $-7.15 \times 10^{8} \mathrm{~Pa}$ for the displacement dependent pressure and $-9.74 \times 10^{8} \mathrm{~Pa}$ for the displacement independent pressure. While Fig. 3(a) shows the axisymmetric radial displacement, the generalized coordinate $w_{1,2}$, corresponding to the buckled shape with $n=2$ circumferential waves, is presented in Fig. $3(\mathrm{~b})$. Results show that the new branch after the pitchfork bifurcation is initially unstable, becoming stable after a folding; this indicates a subcritical buckling, which is more dangerous than supercritical buckling and introduces dynamics with a quick collapse. The axisymmetric axial generalized coordinate $u_{1,0}$ is shown in Fig. 3(c) and the generalized 
a

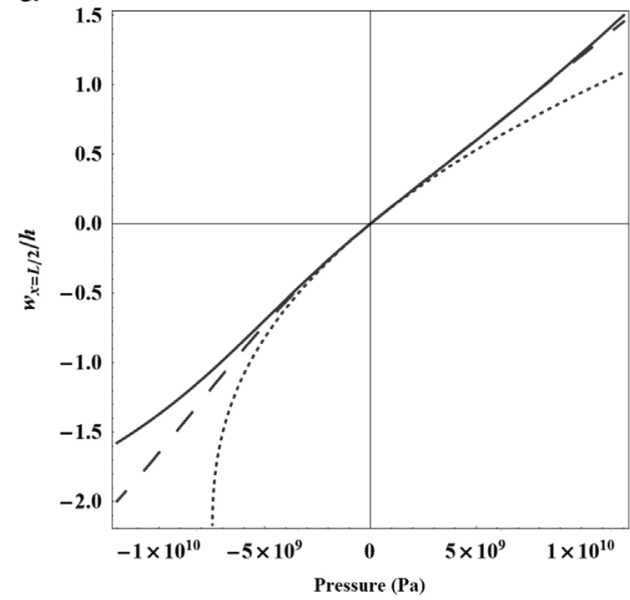

b

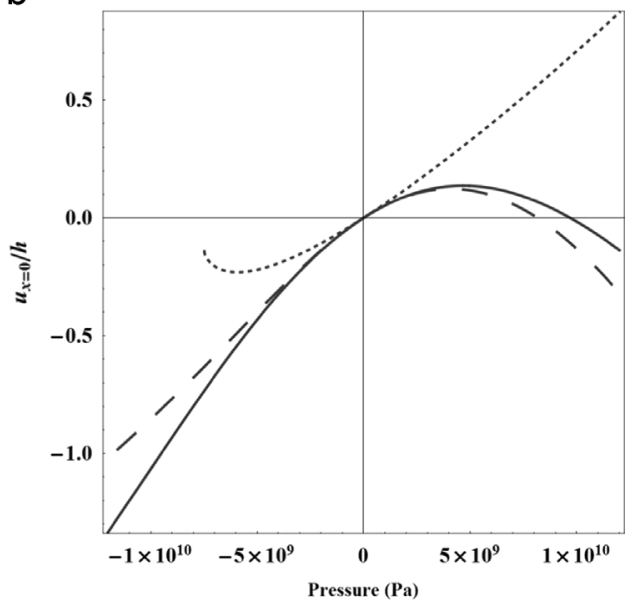

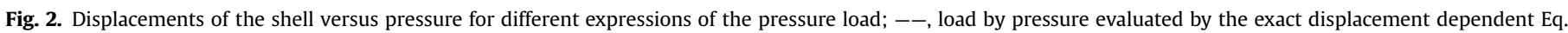

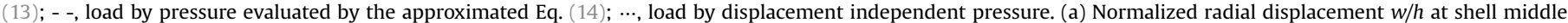
length $x=L / 2$ versus pressure load; (b) normalized axial displacement $u / h$ at the shell edge $x=0$.

a

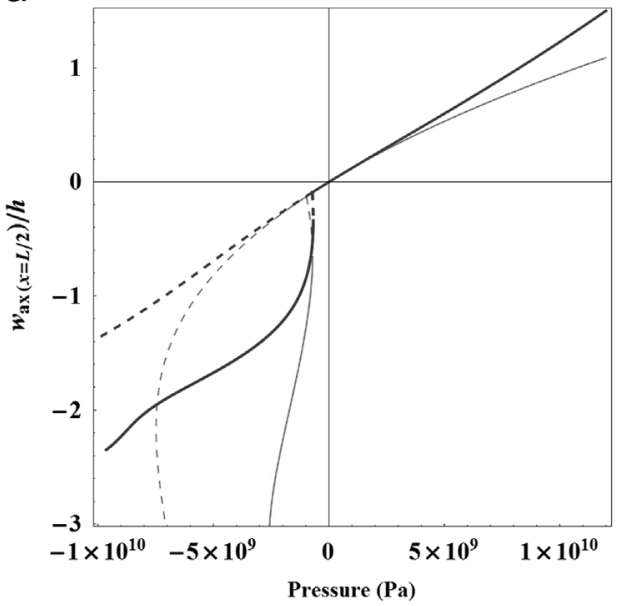

C

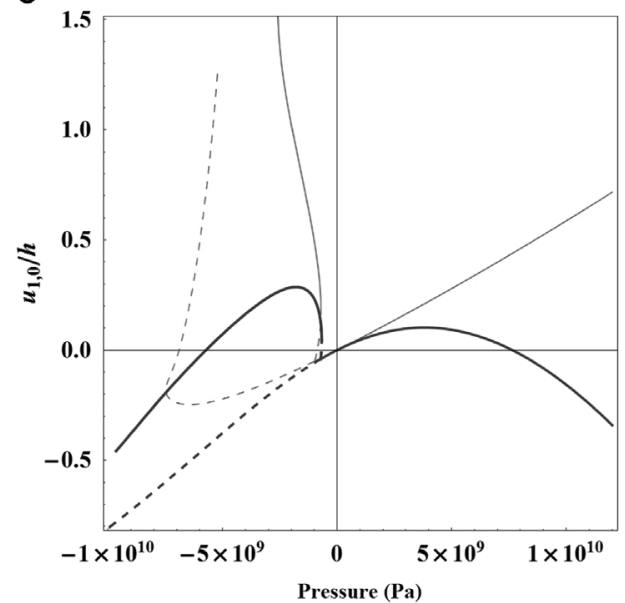

b

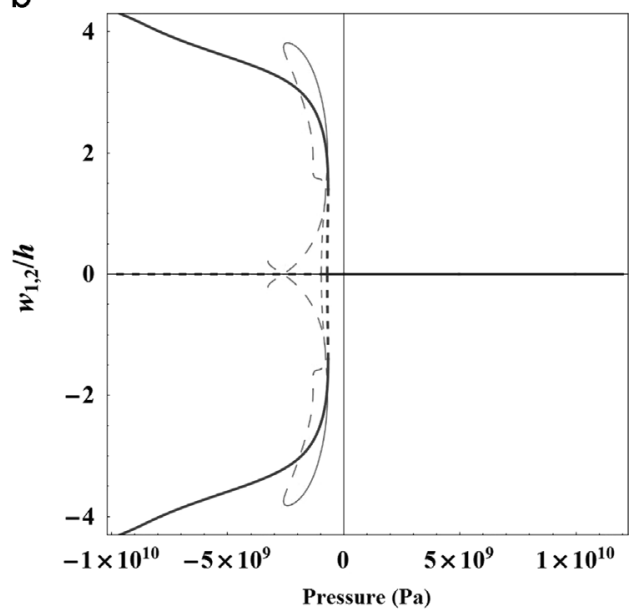

d

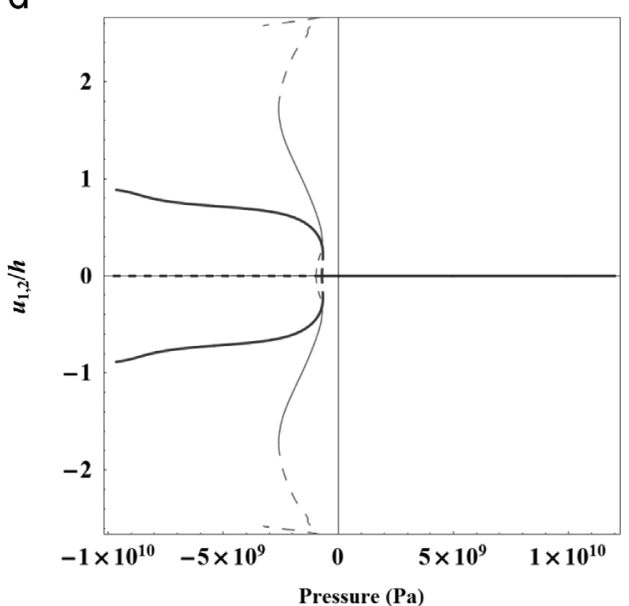

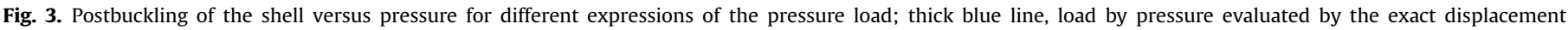

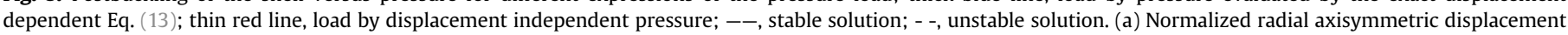
$w / h$ at shell middle length $x=L / 2$ versus pressure load; (b) generalized coordinate $w_{1,2} / h$; (c) generalized coordinate $u_{1,0} / h$; (d) generalized coordinate $u_{1,2} / h$.

axial coordinate $u_{1,2}$ corresponding to the shape with two circumferential waves is shown in Fig. 3(d). Both stable (continuous line) and unstable (dashed line) solutions are plotted; in thick line the displacement dependent pressure solution is presented while the pressure independent solution is shown with a thin line. It is useful to comment that the difference between the two formulations of pressure is here very significant due to the large deformations associated to buckling of a thick shell. For thin shell differences are 

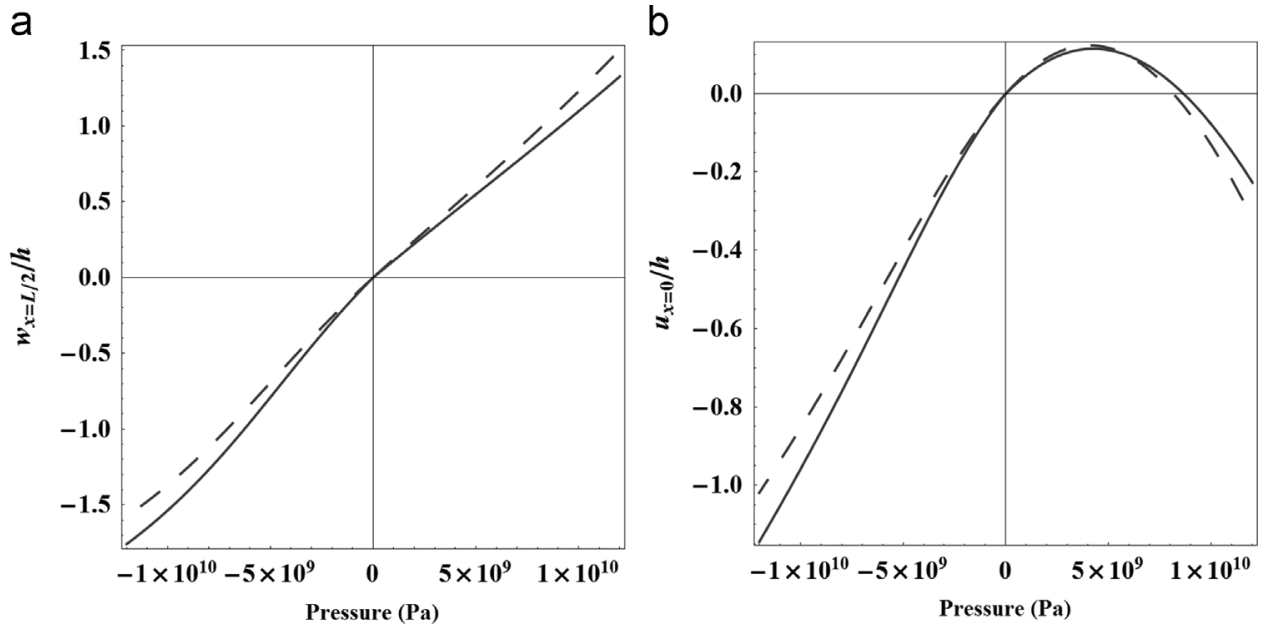

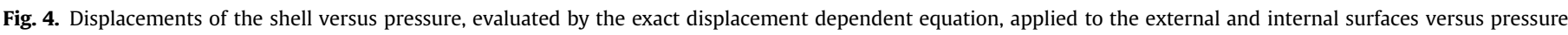

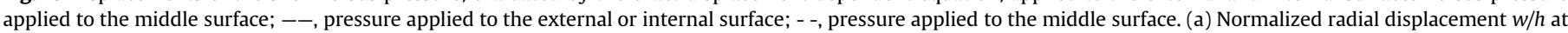
shell middle length $x=L / 2$ versus pressure; (b) normalized axial displacement $u / h$ at the shell edge $x=0$.

smaller since the amplitude of the deformation $w / R$ is smaller for the same order of normalized displacement $w / h$.

Fig. 4 compares the results for pressure applied to the middle surface, as in Figs. 2 and 3, to pressure applied to the internal surface for positive pressure and to external surface for negative pressure. Eq. (32) is used for the pressure applied to the external or internal surface while Eq. (13) is used for the pressure applied to the middle surface. Fig. 4(a) shows the central deflection in normal direction at mid-length. The difference is modest except for very large pressures even if the present shell is quite thick $(R /$ $h=5$ ). It is interesting to observe that a slightly different slope of the curve is obtained at $p=0$ when moving toward positive or negative pressures, due to the switch from internal (smaller area) to external surface (larger area). The axial displacements at the edge $x=0$ are compared in Fig. 4(b).

\subsection{Square plate}

Calculations are done here for a thin simply supported immovable square plate, i.e. with the following boundary conditions: $u=v=w=M_{x}=0$ at $\alpha_{1}=0, a$, and $u=v=w=M_{y}=0$ at $\alpha_{2}=0, b$. The geometrically nonlinear Novozhilov shell theory is used $[28,29,32,33]$, reduced to plate with zero initial curvature. The plate has the following dimensions and material properties: inplane dimensions $a=0.1 \mathrm{~m}, b=0.1 \mathrm{~m}$, thickness $h=0.005 \mathrm{~m}$, Young's modulus $E=2.1 \times 10^{11} \mathrm{~Pa}$, Poisson ratio $\nu=0.3$ (steel). Since the plate is very thin, the pressure is assumed to be applied to middle plane. The expansion used has $\bar{N}=12$ dofs. In particular, in Eqs. (35a)-(35c), [28]

$$
\begin{aligned}
& f_{1}\left(m, \alpha_{1}\right)=f_{2}\left(m, \alpha_{1}\right)=f_{3}\left(m, \alpha_{1}\right)=\sin \left(m \pi \alpha_{1} / a\right), \\
& g_{1}\left(n, \alpha_{2}\right)=g_{2}\left(n, \alpha_{2}\right)=g_{3}\left(n, \alpha_{2}\right)=\sin \left(n \pi \alpha_{2} / b\right) .
\end{aligned}
$$

In expansion (35a)-(35c) only the following 12 terms in the summatories are considered different from zero: $w_{1,1}, w_{1,3}, w_{3,1}$, $w_{3,3}, u_{2,1}, u_{2,3}, u_{4,2}, u_{4,3}, v_{1,2}, v_{1,4}, v_{3,2}, v_{3,4}$.

The deflection of the plate versus pressure for different expressions of the pressure load is presented in Fig. 5(a); the continuous line shows the solution for displacement dependent pressure, while the dashed line is obtained for displacement independent pressure. Since the plate is thin, significant differences between the two formulations are observed for large normal displacements $w$ of about $40 h$. The cross-section of the deformed configuration of the plate for pressure $p=1.5 \times 10^{9} \mathrm{~Pa}$ is shown in Fig. 5(b); Fig. 5(c) presents the deformed configuration for central

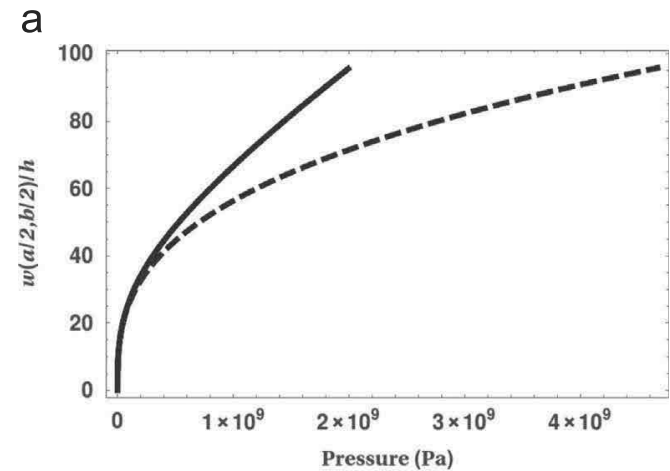

b

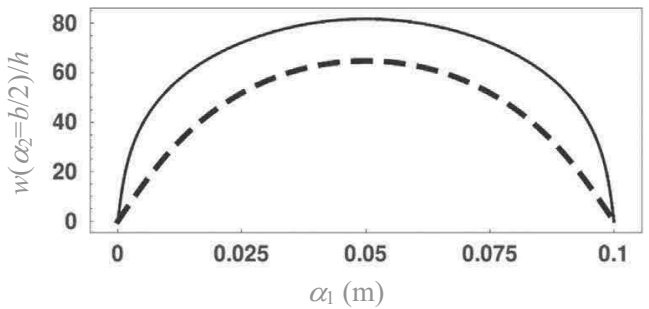

C

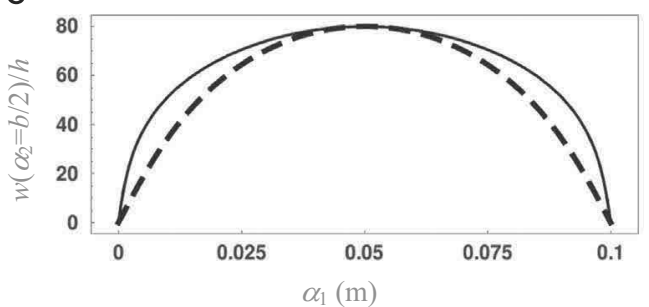

Fig. 5. Deformation of the plate versus pressure for different expressions of the pressure load; --, load by pressure evaluated by the exact displacement dependent Eq. (15); - -, load by displacement independent pressure. (a) Normalized normal displacement $w / h$ at center of the plate versus pressure load; (b) crosssection of the deformed shape of the plate for pressure $1.5 \times 10^{9} \mathrm{~Pa}$; (c) crosssection of the deformed shape of the plate for central deflection of $80 \mathrm{~h}$.

deflection of $80 \mathrm{~h}$. In case of displacement dependent pressure the plate is more bended near the edges; this is due to the rotation of the plate with consequent rotation of the pressure direction that creates this effect. 


\section{Conclusions}

Numerical results show that the exact formulation of the virtual work done by displacement dependent pressure must be used for large deformation of plates and shells. This becomes particularly important for thick shells and plates, especially if made of soft materials. In fact rubbers and biological materials, which can be described with different types of hyperelastic constitutive relationships [33], usually present large strains and large deformations. The formulation can be immediately applied to FGM and laminated composite materials [34,35]. In case of static problems, the additional computational cost of the exact solution is not relevant if compared to the approximate expressions and to the displacement independent pressure.

\section{Acknowledgments}

The author acknowledges the financial support of the NSERC Discovery Grant and Discovery Accelerator Supplement and Canada Research Chairs.

\section{References}

[1] W.T. Koiter, General equations of elastic stability for thin shells, In: Proceedings of the Symposium on the Theory of Shells to honor L.H. Donnell, University of Houston, 1966, pp. 187-227.

[2] A. Libai, J.G. Simmonds, The Nonlinear Theory of Elastic Shells, 2nd edition, Cambridge University Press, New York, USA, 1998 (pp. 308, 412-413).

[3] M.J. Sewell, On configuration-dependent loading, Arch. Ration. Mech. Anal. 23 (1967) 327-351.

[4] H.A. Mang, Symmetricability of pressure stiffness matrices for shells with loaded free edges, Int. J. Numer. Methods Eng. 15 (1980) 981-990.

[5] J.H. Argyris, Sp Symeonidis, Nonlinear finite element analysis of elastic systems under nonconservative loading - natural formulation. Part I. Quasistatic problems, Comput. Method Appl. Mech. Eng. 26 (1981) 75-123.

[6] T.Y. Chang, K. Sawamiphakdi, Large deflections and post-buckling analysis of shell structures, Comput. Method Appl. Mech. Eng. 32 (1982) 311-326.

[7] K. Schweizerhof, E. Ramm, Displacement dependent pressure loads in finite element analyses, Comput. Struct. 18 (1984) 1099-1114.

[8] Y.K. Cheung, D.S. Zhu, Postbuckling analysis of circular cylindrical shells under external pressure, Thin-walled Struct. 7 (1989) 239-256.

[9] D.S. Zhu, Y.K. Cheung, Postbuckling analysis of circular cylindrical shell under combined loads, Comput. Struct. 58 (1995) 21-26.

[10] Z.M. Li, Z.Q. Lin, Non-linear buckling and postbuckling of shear deformable anisotropic laminated cylindrical shell subjected to varying external pressure loads, Compos. Struct. 92 (2010) 553-567.

[11] V.V. Novozhilov, Thin Shell Theory, 2nd edition, Noordhoff, Groningen, The Netherlands, 1964

[12] A. Goetz, Introduction to Differential Geometry, Addison Wesley, Reading, MA, USA, 1970.

[13] E. Carrera, S. Brischetto, M. Cinefra, M. Soave, Effects of thickness stretching in functionally graded plates and shells, Compos.: Part B 42 (2011) 123-133.
[14] A.J.M. Ferreira, E. Carrera, M. Cinefra, C.M.C. Roque, Analysis of laminated doubly-curved shells by a layerwise theory and radial basis functions collocation, accounting for through-the-thickness deformations, Comput. Mech. 48 (2011) 13-25

[15] N. Büchter, E. Ramm, D. Roehl, Three-dimensional extension of non-linear shell formulation based on the enhanced assumed strain concept, Int. J. Numer. Methods Eng. 37 (1994) 2551-2568.

[16] M. Bischoff, E. Ramm, Shear deformable shell elements for large strains and rotations, Int. J. Numer. Methods Eng. 40 (1997) 4427-4449.

[17] M. Bischoff, E. Ramm, On the physical significance of higher order kinematic and static variables in a three-dimensional shell formulation, Int. J. Solids Struct. 37 (2000) 6933-6960.

[18] H. Parisch, A continuum-based shell theory for non-linear applications, Int. J. Numer. Methods Eng. 38 (1995) 1855-1883.

[19] C. Sansour, A theory and finite element formulation of shells at finite deformations involving thickness change: circumventing the use of a rotation tensor, Arch. Appl. Mech. 65 (1995) 194-216.

[20] C. Sansour, W. Wagner, P. Wriggers, J. Sansour, An energy-momentum integration scheme and enhanced strain finite element for the non-linear dynamics of shells, Int. J. Non-linear Mech. 37 (2002) 951-966.

[21] R.A. Arciniega, J.N. Reddy, Tensor-based finite element formulation for geometrically nonlinear analysis of shell structures, Comput. Methods Appl. Mech. Eng. 196 (2007) 1048-1073.

[22] R.A. Arciniega, J.N. Reddy, Large deformation analysis of functionally graded shells, Int. J. Solids Struct. 44 (2007) 2036-2052.

[23] G.S. Payette, J.N. Reddy, A seven-parameter spectral/hp finite element formulation for isotropic, laminated composite and functionally graded shell structures, Comput. Methods Appl. Mech. Eng. 278 (2014) 664-704.

[24] M. Amabili, A new nonlinear higher-order shear deformation theory with thickness variation for large-amplitude vibrations of laminated doubly curved shells, J. Sound Vib. 332 (2013) 4620-4640.

[25] M. Amabili, A non-linear higher-order thickness stretching and shear deformation theory for large-amplitude vibrations of laminated doubly curved shells, Int. J. Non-linear Mech. 58 (2014) 57-75.

[26] F. Alijani, M. Amabili, Non-linear static bending and forced vibrations of rectangular plates retaining non-linearities in rotations and thickness deformation, Int. J. Non-linear Mech. 67 (2014) 394-404.

[27] M. Amabili, Non-linearities in rotation and thickness deformation in a new third-order thickness deformation theory for static and dynamic analysis of isotropic and laminated doubly curved shells, Int. J. Non-linear Mech. 69 (2015) 109-128, http://dx.doi.org/10.1016/j.ijnonlinmec.2014.11.026.

[28] M. Amabili, Nonlinear Vibrations and Stability of Shells and Plates, Cambridge University Press, New York, USA, 2008.

[29] M. Amabili, Comparison of shell theories for large-amplitude vibrations of circular cylindrical shells: Lagrangian approach, J. Sound Vib. 264 (2003) $1091-1125$.

[30] F. Alijani, M. Amabili, Non-linear dynamic instability of functionally graded plates in thermal environments, Int. J. Non-linear Mech. 50 (2013) 109-126.

[31] E.J. Doedel, A.R. Champneys, T.F. Fairgrieve, Y.A. Kuznetsov, B. Sandstede, X. Wang, AUTO 97: Continuation and Bifurcation Software for Ordinary Differential Equations (with HomCont), Concordia University, Montreal, Canada, 1998.

[32] V.V. Novozhilov, Foundations of the Nonlinear Theory of Elasticity, Graylock Press, Rochester, NY, USA, 1953 (now available from Dover, NY, USA).

[33] I.D. Breslavsky, M. Amabili, M. Legrand, Nonlinear vibrations of thin hyperelastic plates, J. Sound Vib. 333 (2014) 4668-4681.

[34] J.N. Reddy, Mechanics of Laminated Composite Plates and Shells: Theory and Analysis, 2nd edition, CRC Press, Boca Raton, FL, USA, 2004

[35] E. Carrera, A study of transverse normal stress effects on vibration of multilayered plates and shells, J. Sound Vib. 225 (1999) 803-829. 